\title{
Development of compression algorithms for hyperspectral aerospace images based on discrete orthogonal transformations
}

\author{
Assiya Sarinova
}

Kazakh Agrotechnical University named after S. Seifullin, 010000, Zhenis avenue 62, Kazakhstan

\begin{abstract}
Annotation. The paper describes the development of compression algorithms for hyperspectral aerospace images based on discrete orthogonal transformations for the purpose of subsequent compression in Earth remote sensing systems. As compression algorithms necessary to reduce the amount of transmitted information, it is proposed to use the developed compression methods based on Walsh-Hadamard transformations and discretecosine transformation.

The paper considers a methodology for developing lossy and highquality compression algorithms during recovery, taking into account which an adaptive algorithm for compressing hyperspectral AI and the generated quantization table has been developed. The conducted studies have shown that the proposed lossy algorithms have sufficient efficiency for use and can be applied when transmitting hyperspectral remote sensing data in conditions of limited buffer memory capacity and bandwidth of the communication channel.
\end{abstract}

\section{Introduction}

Hyperspectral AI remote sensing is necessary for monitoring natural resources and the consequences of emergencies, etc. Currently, the development of software systems for lossy data compression is an urgent task. In solving this problem, there are various areas of research in which research is actively being conducted in the field of developing compression algorithms [1-13].

Algorithms for processing hyperspectral AI with losses based on discrete transformations have been developed. The sequence of stages is as follows.

1. The original transformation of the data structure based on the original hyperspectral AI, storing the values of the coefficients, based on the discrete-cosine transformation with the generated quantization table.

2. Transformation of the data structure based on the original hyperspectral AI, coefficient values, based on Walsh-Hadamard three levels.

3. Transformation of the obtained data structures based on steps $1-2$ by means of the generated coefficient quantization table.

4. Using standard criteria for the quality of restored images.

5. Compression of the obtained structures of stage 4 by one of the standard entropy algorithms.

6. Experimental study of conversion algorithms by compression ratio and quality of recovered data. 


\section{Walsh - Hadamard transformation of three levels}

Consider an example of the Walsh-Hadamard transformation (WHT) for a fragment of hyperspectral AI.

Let the fragment AI represent a matrix consisting of $m$ rows, $n$ columns and $k$ channels: $\mathbf{I}[m$, $n, \mathrm{k}]=\mathbf{I}[10,10,10]$.

The WHT transform is filters that divide the image into low-frequency and high-frequency components. To get the original image, you just need to combine these components again. The direct WHT transformation is represented in matrix form $H_{w t 2}$, a fragment of AI is taken, т.е. $H_{w t 2} \bullet I[m, n, k]=H_{w t 2}{ }^{\prime}[C K]$, где where $\mathrm{SC}$ - spectral component of the matrix $\mathbf{I}[m$, $n, \mathrm{k}]$.

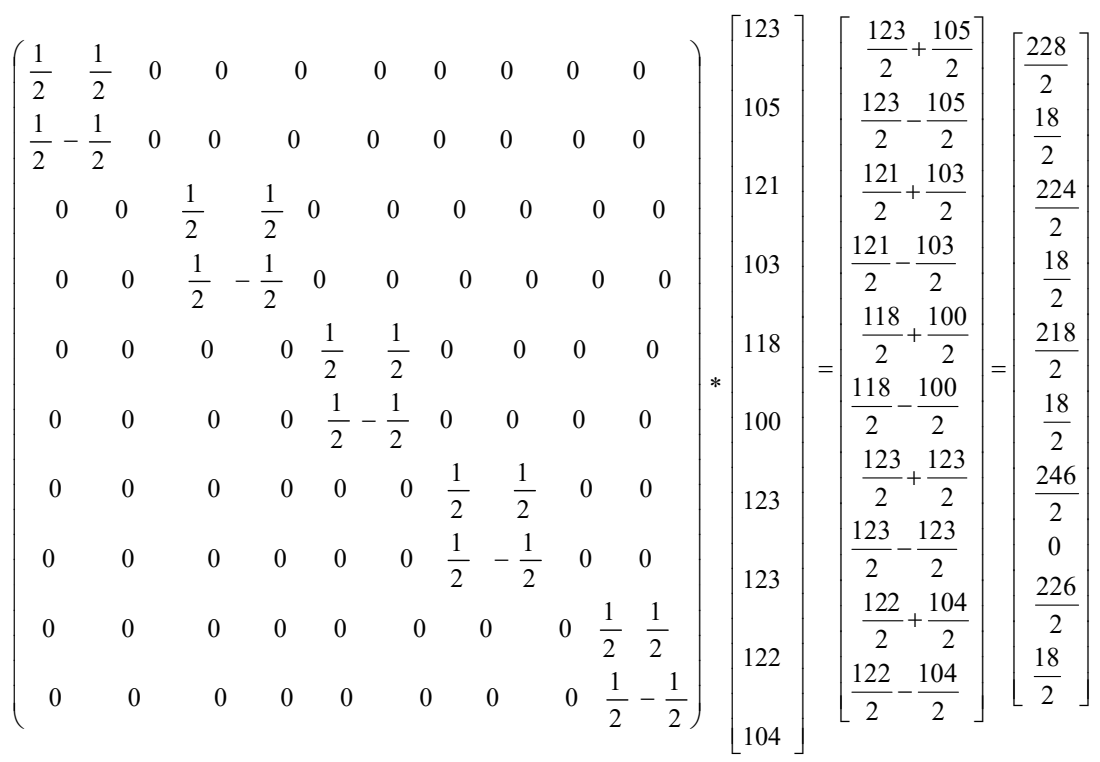

As a result, after such a transformation, we obtain the coefficients of the low-frequency and high-frequency components $\mathrm{SC}=114,9,112,9,114,9,123,0,123$, 9. During quantization, high-frequency coefficients (close to zero and negative values) are rounded to zero. It should be noted that the level matrices $H_{w t 4}$ и $H_{w t 8}$ are calculated in the same way as and $H_{w t 2}$.

At the stage of restoring the original image channels, the SC is decoded. An example of the reverse transformation is presented as follows: 


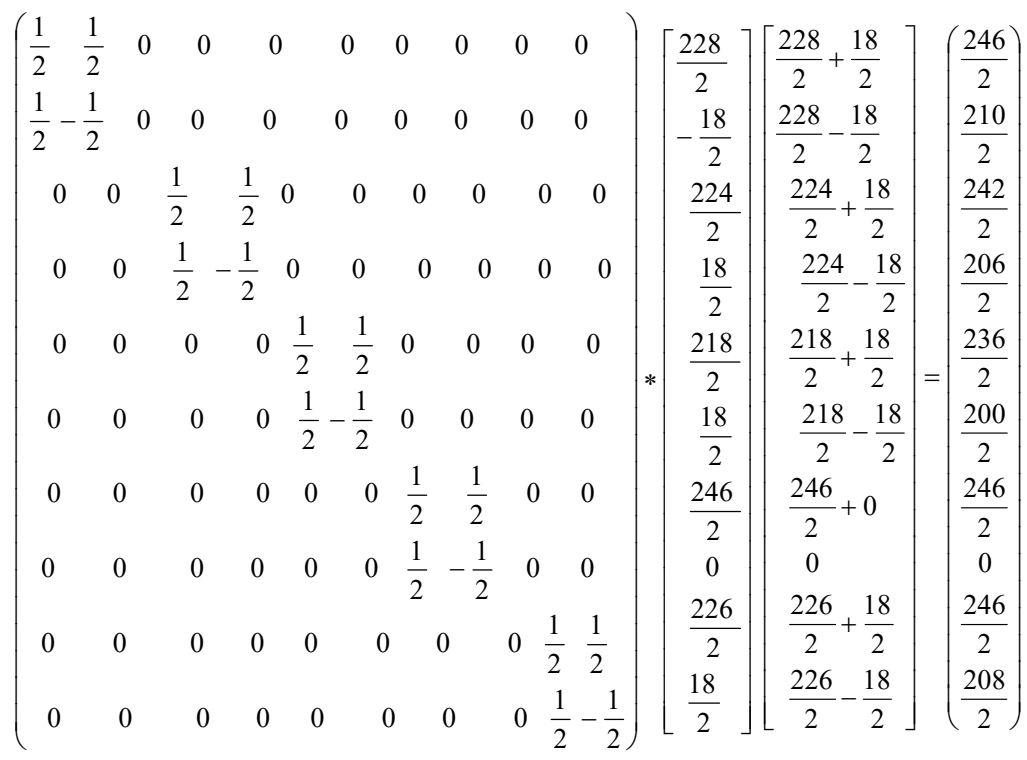

There $H_{w t 2}^{T} \bullet H_{w t 2}{ }^{\prime}[C K]=I[m, n, k]$, where $H_{w t 2}^{T}$ - inverse wavelet transform WHT, $H_{w t 2}{ }^{\prime}[C K]$ - spectral component.

The advantage of the Walsh-Hadamard transformation is an increased ability to detect low-frequency components due to the separation of hyperspectral AI channel regions into sublevels and the generated quantization coefficient.

\section{Discrete-cosine transformation with generation of quantization tables}

The discrete cosine transform (DCT) is a transformation whose linear combination consists of known basis vectors weighted with $\mathrm{n}$ coefficients leading to the original vector.

Let's consider step-by-step transformation of hyperspectral AI channels using DCT.

1. Divide it into s blocks of pixels of size nxn (usually $8 \times 8$ ).

2. Apply PREP to each block, represent each block as a linear combination of 64 basic blocks.

3. All $s$ vectors $W^{(i)}(\mathrm{i}=1,2 \ldots . \mathrm{s})$

4. Generation of the quantization table.

At the first stage, as a result of the DCT conversion, two filters were built - highfrequency and low-frequency. For low-frequency and high-frequency filters, we will introduce some designations: DC is the operator for the low-frequency filter; $\mathrm{AC}$ is the operator for the high-frequency filter. After the DCT transformation, the fragment of hyperspectral AI has the following values (Fig. 1): 


\begin{tabular}{|c|c|c|c|c|c|c|c|}
\hline 40008 & 204 & 708 & -320 & 268 & -139 & -51 & 51 \\
\hline 799 & -176 & 654 & -84 & 60 & 89 & -57 & 114 \\
\hline 188 & -64 & -79 & 102 & -64 & 137 & -55 & 26 \\
\hline 326 & 29 & 157 & -119 & -47 & 79 & -56 & -1 \\
\hline 97 & -94 & 173 & 25 & 44 & 129 & -26 & -63 \\
\hline 33 & 128 & -9 & 118 & -91 & 14 & -96 & -12 \\
\hline-207 & 41 & -198 & 118 & -28 & 34 & 1 & -18 \\
\hline 13 & -1 & -3 & -14 & -2 & -35 & -20 & -11 \\
\hline
\end{tabular}

Fig.1. The coefficients obtained after the DCT conversion

It can be seen from Fig. 1 that the high values of the conversion coefficients are concentrated in the upper left corner and only a small number of low-frequency coefficients prevail over the rest. This allows you to reduce the values at the next stages of compression.

The second stage is the process of generating quantization tables. Quantization is performed in this way:

1. The arithmetic mean $\mathbf{I}_{\mathrm{av}}$ was calculated from all the values of the file.

2. To select the numerical quantization index Quant, the number for quantization was calculated, Quant $=$ quantValue $\times \mathbf{I}_{a v} / 100$. For example, the numerical quantization index: $\mathbf{I}_{\mathrm{av}} * 95 \%=285=$ Quant. At the same time, the DC coefficients remain intact. For each hyperspectral AI channel, its own quantization table is generated.

For the above fragment of hyperspectral AI, the matrix after quantization is shown (Fig.2). The quantization process is key in the compression process, the advantage of representation in the frequency domain lies in the visual quality of the reconstructed images. As a result, after quantization, most of the coefficients are zero.

$\begin{array}{llllllll}40008 & 0 & 708 & -320 & 268 & 0 & 0 & 0 \\ 799 & 0 & 654 & 0 & 0 & 0 & 0 & 0 \\ 0 & 0 & 0 & 0 & 0 & 0 & 0 & 0 \\ 326 & 0 & 0 & 0 & 0 & 0 & 0 & 0 \\ 0 & 0 & 0 & 0 & 0 & 0 & 0 & 0 \\ 0 & 0 & 0 & 0 & 0 & 0 & 0 & 0 \\ 0 & 0 & 0 & 0 & 0 & 0 & 0 & 0 \\ 0 & 0 & 0 & 0 & 0 & 0 & 0 & 0\end{array}$

Fig. 2. Quantization of the DCT coefficients

As a result, most of the zeros will be placed at the end of the data compression stream. This stream with many consecutive zeros at the end of the block is optimized to achieve high compression in the entropy coding of the adaptive Huffman algorithm. 


\section{Experiments of the developed algorithms of lossy transformations}

To determine the effectiveness of the proposed adaptive transformation algorithms in terms of the degree of compression and quality of the recovered images, as well as the limits of its applicability, a number of experiments were conducted on hyperspectral AI (Aviris remote sensing system), (Fig.3). The experiments were performed on a PC with an IntelCore i 5 processor $2.5 \mathrm{GHz}$ and $4 \mathrm{~GB}$ RAM running Windows 8.1 operating system.

Indicators of compression degrees in terms of the number of channels of orthogonal Walsh-Hadamard transformations, DCP and JPEG 2000 Lossy are shown in Fig. 3. It is shown that DCT prevails over the Walsh-Hadamard and JPEG Lossy transformations in the compression ratio with high quality of the restored images. As can be seen from Fig.3, the indicators of the degrees of compression of the DCT with losses are superior in the degree of compression of the Walsh-Hadamard transformation and the JPEGLossy compressor.

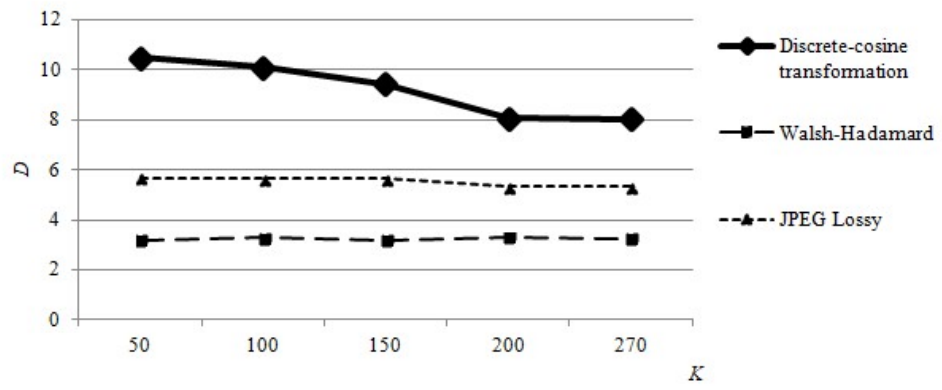

Fig. 3. Dependencies of R on D

Indicators of the quality metrics of the restored images were determined using PSNR and $S K O$. The degree of distortion compares the ratio between compression and distortion in lossy algorithms. The score is defined as the average of the number of bits needed to represent each pixel. Measured in bits per pixel (bpp - bits per pixel). Distortion is usually measured using PSNR. Figure 4.28 shows the dependence of PSNR on the compression ratio $D$. The following Figure 5 shows the dependence of PSNR on $D$ for $D C T$ and the Walsh-Hadamard transformation.

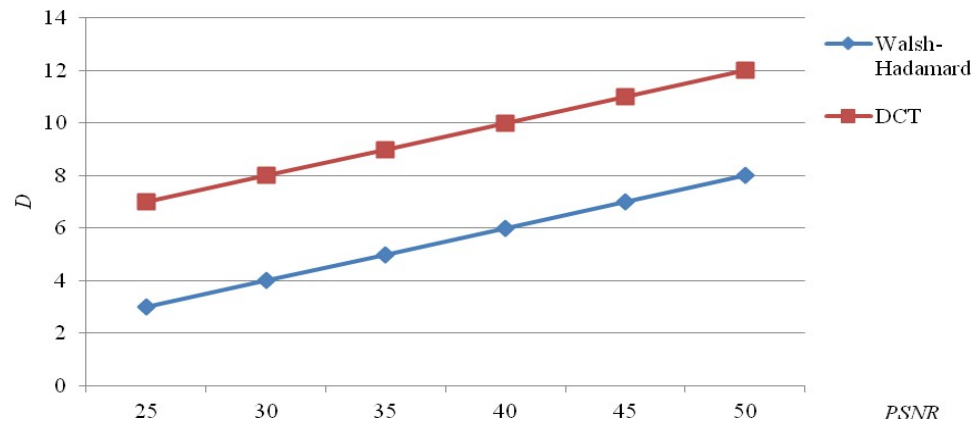

Fig. 4. PSNR dependence on For Walsh-Hadamard and DCT 


\section{Conclusion}

Based on the above results of the compression experiment based on orthogonal and wavelet transformations of hyperspectral AI with losses, it can be concluded that the developed mathematical and compression software, to some extent occupies a leading position in the degree of compression and quality of the restored images, depending on the selected preparatory processing, significantly demonstrates and surpasses analogues on all test sets. Based on the conducted research, the following conclusions should be drawn:

- the development of algorithms based on discrete transformations makes it possible to increase the compression ratio to $(\mathrm{R}=12)$;

- the proposed lossy approach is defined in adaptive transformations based on the Walsh-Hadamard transformation, discrete-cosine transformation and the generated quantization table and subsequent adaptive coding.

\section{Литература:}

1. D. S. Sujithra, T. Manickam, D. S. Sudheer. Compression of hyperspectral image using discrete wavelet transform and walsh hadamard transform. International Journal of Advanced Research in Electronics and Communication Engineering. Vol. 2(3), 314 - 319 pp. 2013.

2. P. Keerthana, A. Sivasankar. The impact of lossy compression on hyperspectral data adaptive spectral unmixing and PCA classification. International Journal of Science and Modern Engineering, Vol.1(7), 35-37 pp. 2013.

3. Poonam and R.S. Chauhan. Compression and Classification of Hyperspectral Images using an Algorithm based on DWT and NTD. Advance in Electronic and Electric Engineering. Vol.3(4). 447-456 pp. 2013.

4. L. Santos, E. Magli, R. and et. Lossy hyperspectral image compression on a graphics processing unit: parallelization strategy and performance evaluation. Journal of Applied Remote Sensing. Vol. 7, 074955-1-15 pp. 2013.

5. S.M. Ramesh, P. Bharat, J. Anand, J. AnbuSelvan. Analysis of Lossy Hyperspectral Image Compression Techniques. International Journal of Computer Science and Mobile Computing, Vol.3(2), 302-307 pp. 2014.

6. A. Sarinova, A. Zamyatin. Hyperspectral regression lossless compression algorithm of aerospace images. E3S Web Conferences 149, 02003 (2020).

7. S. Kudubayeva, N. Amangeldy, A. Sundetbayeva and A. Sarinova. ACM International Conference Proceeding Series 8, (2019). (2015).

8. A. Sarinova, A. Zamyatin and P. Cabral DYNA (Colombia) 82 (190), 166-172

9. A. V. Neftissov, O. A. Andreyeva, Sarinova A. Zh., Investigation of the properties of reed switches in devices for resource-saving relay protection of the electrical part of power plants. AIP Conference Proceedings 2337, No. 030010, 2021.

10. A. Zh. Sarinova, A. V. Drobinsky, L. N. Kirichenko, Using and applying computer technologies for studying transient processes in electrical circuits. AIP Conference Proceedings 2337, No. 030013, 2021.

11. Sarinova A., Zamjatin A., Methodology for Developing Algorithms for Compressing Hyperspectral Aerospace Images used on Board Spacecraft, E3S Web of Conferences 223, 02007, 2020.

12.S. Abimuldina, A. Sarinova, L. Sarlybayeva and N. Akhmetova. DYNA (Colombia) 84 (202), 289-294 (2017). 
13. A. Sarinova, A. Zamyatin and P. Cabral. Lossless compression of hyperspectral images with pre-byte processing and intra-bands correlation. DYNA (Colombia) 82 (190), 166-172 (2015). 